Les dictionnaires bilingues des maîtres ou professeurs auteurs de manuels pédagogiques

\title{
Un intellectuel exilé dans la tourmente du Risorgimento : Antonio Ronna, lexicographe et professeur
}

Francesco Paolo Alexandre Madonia

\section{OpenEdition}

Édition électronique

URL : https://journals.openedition.org/dhfles/3937

DOI : $10.4000 /$ dhfles.3937

ISSN : 2221-4038

Éditeur

Société Internationale pour l'Histoire du Français Langue Étrangère ou Seconde

Édition imprimée

Date de publication : 1 juin 2016

Pagination : $51-73$

ISSN : 0992-7654

Référence électronique

Francesco Paolo Alexandre Madonia, «Un intellectuel exilé dans la tourmente du Risorgimento : Antonio Ronna, lexicographe et professeur ", Documents pour l'histoire du français langue étrangère ou seconde [En ligne], 56 | 2016, mis en ligne le 04 septembre 2017, consulté le 25 mars 2023. URL : http://journals.openedition.org/dhfles/3937 ; DOI : https://doi.org/10.4000/dhfles.3937

Ce document a été généré automatiquement le 25 mars 2023.

Tous droits réservés 


\title{
Un intellectuel exilé dans la tourmente du Risorgimento : Antonio Ronna, lexicographe et professeur
}

\author{
Francesco Paolo Alexandre Madonia
}

\section{Introduction}

1 Les dictionnaires d'Antonio Ronna connurent un grand succès au XIX ${ }^{\mathrm{e}}$ siècle, mais la renommée de leur auteur n'est pas liée uniquement à ses travaux lexicographiques. Ce professeur qui partagea sa vie à la fois aventureuse et studieuse entre la France et l'Italie, fut aussi l'un de ces intellectuels italiens qui, fuyant le régime autrichien, vinrent se réfugier en France avant que l'Unité nationale ne s'accomplisse.

2 Pour mieux comprendre l'influence du Risorgimento sur la vie d'exilé que Ronna mena, nous retracerons dans la première partie de cet article les éléments biographiques de l'auteur, jusqu'à présent méconnus; dans la deuxième et troisième parties, nous examinerons ses manuels et ses dictionnaires, afin de vérifier s'il existe des liens entre ces ouvrages à vocation pédagogique, sans négliger de mettre en évidence les raisons qui en déterminèrent le succès ou l'échec à une époque où la lexi-cographie bilingue franco-italienne atteint un public de plus en plus large.

\section{Biographie et cadre historique}

3 Antonio Ronna naît à Crema le 8 janvier 1801. Son père Antonio est un imprimeur ; sa mère Anna Frigerio meurt lorsqu'il est en bas âge (Provenzal 1867: 328-337). D'une intelligence particulièrement précoce, à quatorze ans il est admis exceptionnellement à l'Université de Pavie, à la Faculté de droit. En mars 1821, Antonio Ronna, sur le point d'obtenir la licence, abandonne ses études pour participer aux mouvements libéraux piémontais; à cette époque, Victor-Emmanuel $\mathrm{I}^{\mathrm{er}}$ abdique en faveur de son frère Charles-Félix et nomme en tant que régent le jeune Charles-Albert avant de s'exiler à 
Nice. Ronna se met alors à la disposition du gouvernement provisoire, d'autant que Charles-Albert prône une Constitution inspirée à celle de l'Espagne de 1812 (La Pepa). Charles-Albert ayant renoncé à ses projets constitutionnels, Ronna, déçu par ce revirement, obtient avec 34 étudiants lombards qui s'étaient insurgés avec lui, de s'embarquer pour la Catalogne; là il combat aux côtés de l'Empecinado, le plus célèbre des guerrilleros, contre l'absolutisme de Ferdinand VII, allié aux Français (Bonola 1869 : 120). En novembre 1822, après une bataille dans laquelle il est blessé à une main, il est promu au grade de lieutenant. Le 15 janvier 1823 il est mis à l'ordre du jour pour acte de valeur; mais une semaine plus tard il est fait prisonnier par George Bessières ${ }^{1}$. Condamné à être passé par les armes avec quatre autres compagnons, il est conduit sur le lieu de l'exécution mais il réussit à s'enfuir. Après la victoire des royalistes, Ronna se réfugie à Londres. En 1826, il publie un dictionnaire anglais-français-italien, sa première œuvre lexicographique. En 1827, il se marie avec Mary Pilton. En 1831, il abandonne l'Angleterre et sa famille pour s'unir aux insurgés, quand il apprend que les espoirs italiens ont été une fois de plus déçus. Il s'établit à Paris (Fonzi Columba 1972 : 447-449), où il occupe une fonction centrale dans les initiatives du libraire éditeur parisien Baudry, comme le démontre le fait qu'il lui confie la direction de deux collections littéraires importantes (cf. § 3.2.2). En 1836, Ronna poursuit et complète, grâce aux fiches laissées par Biagioli, le Dictionnaire italien-français, français-italien (Colombo $2007: 250$ ). À l'activité de lexicographe, Ronna joint celle d'enseignant: dans l'antique collège royal Chaptal, où il occupera la chaire d'italien pendant vingt-deux ans, puis à l'école communale François I ${ }^{\text {er }}$. En 1848, les événements des Cinq journées de Milan l'incitent à rentrer dans sa patrie, où le gouvernement provisoire de la Lombardie veut lui confier une charge publique, mais il en est empêché par des exigences familiales. Dans les dix années suivantes qui voient encore échouer les aspirations du Risorgimento, des préposés à l'instruction publique du Piémont lui demandent à plusieurs reprises aides et conseils. C'est pendant cette période qu'il écrit deux rapports sur des livres éducatifs d'Angleterre et de France, dont le dernier avait été composé sur l'invitation du ministre Francesco De Sanctis. En 1859, après la campagne d'Italie qui voit s'affronter les armées franco-piémontaise et autrichienne, Ronna revient définitivement en Italie, où le comte Pepoli, ministre de l'agriculture et du commerce, le charge de fonder et de diriger l'Institut technique de Palerme. Mais Ronna tolère mal le climat sicilien et obtient du ministre Torelli la charge de proviseur à l'Institut de Marine marchande à Livourne, où il conclura sa carrière (Colombo 2007 : 250-251n). Après l'expédition des Mille, les Bourbons perdent définitivement le royaume des Deux-Siciles et Garibaldi entre à Naples le 7 septembre 1860. Le $1^{\text {er }}$ janvier 1861, Cavour décore Ronna de la croix de «S.S. Maurizio e Lazzaro ». Le 17 mars 1861 Victor-Emmanuel II prend le titre de roi d'Italie. En 1866, la troisième guerre d'indépendance italienne est proclamée : l'Italie signe l'armistice avec l'Autriche le 12 août 1866. Antonio Ronna a pu voir sa patrie unifiée lorsqu'il meurt à Livourne le 16 septembre 1866. Il est enterré dans le cimetière d'Antignano².

\section{Les manuels pédagogiques}

4 L'activité intellectuelle d'Antonio Ronna est intense et variée et se poursuit tout au long de sa vie. Dès sa jeunesse il s'adonne à l'écriture poétique et à la prose avec un conte tiré des chroniques d'Espagne (Provenzal 1867: 329); en 1862, il écrit un recueil de Fables. Par ailleurs, il s'est consacré à la diffusion de la littérature italienne en France 
grâce à la publication d'anthologies ${ }^{3}$ et d'un essai de critique littéraire sur Giusti ${ }^{4}$. Sa condition d'exilé le rend polyglotte : il a une excellente connaissance de l'espagnol, de l'anglais et du français; ce sont ces compétences qui détermineront sa vocation de lexicographe et de pédagogue.

\subsection{Le Guide de la conversation français-italien}

Le Guide de la conversation français-italien, à l'usage des Voyageurs et des Étudiants, par A. Ronna, comprenant un vocabulaire des mots usuels, des conjugaisons appliquées, des phrases familières et élémentaires, des dialogues, des idiotismes, proverbes, des modèles de lettres, billets, la concordance des monnaies, poids et mesures de France et d'Italie est le premier ouvrage pédagogique de Ronna. Publié à Paris, chez Hingray (cf. § 3.2.1), le Guide, dont nous analyserons la version bilingue français-italien, existe aussi en six langues (français, anglais, allemand, italien, espagnol, portugais), dont sont coauteurs Léo Smith (anglais), Édouard-Marie-Emmanuel Adler-Mesnard (allemand), Eugenio de Ochoa (espagnol) et le P. José Ignacio Roquette (portugais) ; il existe aussi une édition quadrilingue (anglais, français, allemand, italien) et trilingue (françaisanglais-italien), ainsi que d'autres versions bilingues toujours avec les co-auteurs mentionnés ci-dessus.

6 Nous avons dénombré huit éditions du Guide (1840, 1843, 1853, 1855, 1859, 1861, 1864, 1873) que nous avons consultées à la BNF; d'autres éditions pourraient néanmoins exister vu que l'ouvrage connut un très grand succès. Le Guide comporte plusieurs parties : un Vocabulaire organisé par thèmes (Le monde visible, Le monde invisible, Les corps célestes...); les Nombres (cardinaux, ordinaux, collectifs, fractionnaires, proportionnels, de répétition); la Conjugaison (les verbes sont conjugués avec un substantif : «J'ai une maison; Tu as une chambre » etc.) ; des Phrases élémentaires ; des Conversations ; des Lettres et billets ; les Proverbes et idiotismes ; les Monnaies.

7 La nomenclature ne change pas au fil des éditions; elle est ordonnée alphabétiquement à l'intérieur de chaque section thématique et contient des unités lexicales simples, comme il se doit pour un vocabulaire. La seule différence entre les diverses éditions concerne les tables; en 1853, par exemple, paraissent deux tables supplémentaires: Monete degli Stati Pontificali et Monete del Regno di Napoli ${ }^{5}$.

8 L'Avis de l'éditeur qui, dans les éditions de 1840 et 1843, annonce la création de la «Collection Polyglotte » et qui présente l'avantage d'être «aussi utile aux voyageurs qu'aux étudiants ", renforce, dès 1855 , la double vocation du Guide, se réclamant de "premiers essais de Poppleton, Bellenger, Hamonière » : vocation pédagogique, car il permet d'acquérir "par une application pratique, les règles les plus difficiles de la grammaire $»^{6}$, et pratique "en faisant donner un plus grand développement aux dialogues concernant les chemins de fer et les découvertes les plus récentes ». Le Guide s'insère ainsi dans la tendance à l'enseignement/apprentissage multilingue qui caractérise le contexte européen de la première moitié du XIX ${ }^{e}$ siècle (Rius Dalmau 2009 : 142-144). Par ailleurs, le public visé reste aussi large que possible : « Il y a dans ces petits volumes économiques des matières utiles pour toute l'échelle de la société, de l'humble travailleur à l'homme d'État, de l'homme du monde au grave philosophe ». Les autres éléments paratextuels comprennent les abréviations françaises divisées en orthographe (article, adjectif, pronom, préposition, conjonction, négation), écriture, et 
impression ; les abréviations italiennes ; des Tables des monnaies, poids et mesures. En dépit de la présence d'informations grammaticales, la prononciation n'est pas indiquée.

En ce qui concerne les aspects formels, la lisibilité et la consultabilité sont assez bonnes ; les différentes sections du vocabulaire sont divisées par des espaces blancs ; il existe deux formats : in- $16^{\circ}$, vendu à 2 francs 50 , et in- $32^{\circ}$, vendu à 1 franc $50^{7}$.

Enfin, il importe de remarquer que ni les abréviations, ni les conjugaisons, ni la nomenclature ne correspondent aux parties homonymes présentes dans les différentes éditions des dictionnaires dont Ronna est le co-auteur ou l'auteur.

\subsection{Lectures italiennes}

11 En 1841, Ronna publie Lectures italiennes, un cours supérieur comprenant: Tasse, Jérusalem délivrée chants XI et XII, Dante, premier et troisième chants de l'Enfer, Manzoni, Inni sacri. Il s'agit de la reprise d'un travail de Niccolò Giosafatte Biagioli (1769-1830), illustre spécialiste de Dante, que Ronna complète. L'Avis au lecteur nous permet en effet d'attribuer à Ronna le commentaire au Tasse et à Manzoni, alors que les notes à l'Enfer sont de Biagioli. L'ouvrage fut adopté par le Conseil royal de l'Université de Paris le 27 juillet 1841.

\subsection{Les Novelline per uso de' fanciulli e de' giovinetti}

Les Novelline per uso de' fanciulli e de' giovinetti, publiées en 1853, présentent un statut hybride, car sans avoir d'intention explicitement pédagogique, elles contiennent des notes lexicales en bas de page («monelli-gamins»; «spiccioli - changer en menue monnaie ", etc.) qui semblent destiner l'ouvrage à un public de jeunes apprenants français.

\section{Les dictionnaires}

13 Le corpus que nous analysons comprend l'ensemble des dictionnaires bilingues français-italien et italien-français dont Ronna a été le co-auteur ou l'auteur ${ }^{8}$. Il est possible de dénombrer trois différents ouvrages lexicographiques, dont chacun comporte plusieurs rééditions. Puisqu'il s'agit de dictionnaires à vocation prioritairement scolaire, nous analyserons en particulier les éléments qui caractérisent cette typologie d'ouvrage : le prix, le poids, le traitement des mots triviaux, la place faite à la grammaire (Lillo 2006 : 230-232).

\subsection{Les éditions des dictionnaires}

14 Antonio Ronna est l'auteur de trois dictionnaires, dont les deux premiers, publiés en 1836, constituent la reprise de travaux lexicographiques précédents, notamment le Dictionnaire français-italien de Niccolò Giosafatte Biagioli (cf. § 2.2) et le Dictionnaire français-italien de Jean-Philippe Barberi. Ronna ne publiera son propre dictionnaire qu'en 1858.

15 Parmi les différentes éditions de ces trois ouvrages, le Biagioli-Ronna représente sans conteste celui qui a rencontré le plus grand succès : ses éditions s'échelonnent en effet, 
du vivant de l'auteur, de 1836 à 1860, mais les réimpressions se succèdent jusqu'à 1897 . Le succès de cet ouvrage tient d'une part à la renommée du premier auteur, de l'autre à son entrée dans le marché à une époque où le public est friand de dictionnaires de poche qui constituent une innovation face au modèle représenté par le dictionnaire d'Alberti (Madonia 2016: 172-173). Approuvé par le Conseil royal de l'Université de Paris dès la première édition, le Biagioli-Ronna s'améliore au fil des années par l'ajout de pages consacrées à la théorie des verbes italiens, ainsi que de LVI pages consacrées à leur conjugaison (édition de 1838).

16 Le Barberi-Ronna compte cinq éditions, toutes identiques à la première. Le succès de cet ouvrage a été très limité, sans doute à cause de sa très médiocre lisibilité (cf. § 3.4) et de l'absence d'explications grammaticales (cf. § 3.5)

Dictionnaire italien-français de Ronna compte quatre éditions du vivant de l'auteur plus deux sans date. Tous les exemplaires sont quasiment identiques. Le Ronna s'inspire beaucoup du Biagioli-Ronna et cherche à en égaler les qualités sans toutefois y parvenir complètement, car sa prétention d'être à la fois un dictionnaire scolaire et un dictionnaire général brouille quelque peu les attentes du public (cf. § 3.3.3).

\subsection{Les éditeurs}

18 La plupart des éditions du dictionnaire Biagioli-Ronna sont publiées par Charles Hingray, né à Épinal en 1796 (Munier 2003 : 177). Hingray, nommé au barreau en 1820, s'engage dans le parti libéral sous la Restauration. Après 1827 , il ouvre une librairie à Paris. Radical sous le règne de Louis-Philippe, il est élu colonel de la $\mathrm{X}^{\mathrm{e}}$ légion de la garde nationale (Martin, Chartier, Vivet 1985: 174) après la proclamation de la République : c'est sans doute la foi dans les idéaux républicains qui a constitué le lien d'amitié entre lui et Ronna, qui s'était établi à Paris justement au moment de la monarchie de Juillet. Dans les années 1860, il vend sa librairie à Fouraut et fils qui continueront à imprimer le dictionnaire de Ronna. Charles Hingray s'éteint à Paris le 7 juin 1870 .

19 L'édition vénitienne (1839-1840) est publiée par Giuseppe Antonelli (1793-1861) qui avec son fils Antonio (1817-1889) fonde une maison d'édition figurant parmi les plus importantes de l'époque et qui publie d'innombrables ouvrages dont le plus célèbre est La nuova biblioteca degli scrittori latini, de 1848 (Dizionario biografico degli italiani 1961, vol. 3 : 497-498). Enfin, l'édition napolitaine (1841) est sans nom d'éditeur.

20 Le dictionnaire Barberi-Ronna est publié par Louis-Claude Baudry, habile libraire imprimeur, qui réussit à gagner une réputation internationale grâce à ses reproductions, souvent non autorisées mais prestigieuses, d'auteurs italiens et anglais (Palazzolo 1987 : 209-210). En correspondance avec Vieusseux, il contribue à la diffusion de la culture italienne en France, grâce au lien qu'il entretient avec des réfugiés de l'envergure de Tommaseo (Palazzolo 1987: 214-217). Après 1840, les lois sur la reproduction des œuvres littéraires étant devenues plus sévères, Baudry se convertit à l'édition scolaire; c'est à cette époque qu'il confie à Ronna la direction d'importantes collections: la «Collezione de'migliori autori italiani antichi e moderni» et la «Biblioteca poetica italiana».

21 Le dictionnaire de Ronna est publié chez Hingray ( $c f$. $\S 3.2 .1$ ). L'édition de 1883 est publiée à Naples chez l'éditeur Francesco Casella. La Librairie Casella, spécialisée à l'origine en cartes et instruments nautiques, fut fondée par Gennaro Casella en 1825. 
Après l'Unité italienne, Casella se tourna vers l'activité d'édition, que continua son fils Francesco ; ce dernier développa d'un côté le commerce de livres anciens et de l'autre continua l'activité d'édition, même scolaire, qu'il poursuivit jusqu'à la réforme Gentile (Cristiano $1986: 86-87)$.

\subsection{Typologies}

Les trois dictionnaires dont Ronna est le co-auteur ou l'auteur, constituent des ouvrages qui présentent les caractéristiques typiques des dictionnaires d'apprentissage au XIX ${ }^{\mathrm{e}}$ siècle : format de poche, un seul volume dans la plupart des cas, définitions nominales, taille de la police diminuée (Pruvost 2001 : 74-75). Les avis de l'éditeur ou la préface de Ronna fournissent des indications précises concernant la typologie de ces dictionnaires.

Dans l'avant-propos du Biagioli-Ronna, on lit que ce dictionnaire s'adresse à » [...] la jeunesse, à laquelle nous dédions particulièrement cet ouvrage ». En ce qui concerne les objectifs, Ronna déclare toujours dans son avant-propos que : « tous nos efforts ont eu pour objet d'aider dans ses recherches la jeunesse studieuse ». C'est dans ce but, et dans la limite offerte par un dictionnaire abrégé, qu'ont été fournies les explications grammaticales, les tournures choisies, les définitions de mots relatifs à l'histoire, aux sciences, aux arts. Le Biagioli-Ronna est conçu pour un public français : les LVI pages consacrées à la conjugaison des verbes italiens sont rédigées en français; en outre, les indications sur la prononciation concernent l'italien; enfin, la partie intitulée «Dictionnaire géographique italien-français » contient une précision absente dans la partie équivalente français-italien: "Les noms des villes se terminant par - $a$ sont toujours féminins; mais s'ils se terminent par une autre voyelle, on peut utiliser les deux genres ".

Dans l'avant-propos du Barberi-Ronna, l'auteur précise que ce dictionnaire général s'adresse à un public scolaire: «[...] ce petit dictionnaire que nous offrons aux étudiants »; Barberi précise par ailleurs, toujours dans l'avant-propos :

Nous avons cru pouvoir rendre service aux étudiants, en entreprenant le travail d'un dictionnaire très-portatif, auquel nous avons donné cependant une étendue qui est, pour le moins, le double de celle des autres dictionnaires dits de poche, en y réunissant, d'une manière claire, le plus de choses utiles aux étudiants [...].

À la différence du Biagioli-Ronna, le Barberi-Ronna n'est pas spécialement conçu pour un public français : la liste des abréviations est fournie dans les deux langues, de même que la table des noms propres de personnes, de pays, de nations. Toutes les indications fournies après l'entrée (catégorie grammaticale, genre, nombre, marques et éventuelles gloses explicatives ou de définition) sont données en français dans la partie italienfrançais et en italien dans la partie français-italien.

Quant au dictionnaire de Ronna, comme nous l'avons déjà remarqué, c'est un dictionnaire qui se veut à la fois scolaire et général : dans l'avis de l'éditeur on lit que son but est de "faciliter les études, [et] venir en aide aux voyageurs »; et d'ajouter : "Ainsi par la richesse de sa nomenclature, par les explications grammaticales, scientifiques et artistiques qu'il donne chaque fois qu'elles sont nécessaires, il peut, dans un cadre restreint, remplacer pour les termes qu'il y a fait entrer, le secours d'un plus grand Dictionnaire». 
Enfin, à l'instar du Biagioli-Ronna auquel l'apparentent bien des points, le Ronna est conçu pour un public français: les indications contenues dans le dictionnaire géographique sont les mêmes que celles du Biagioli-Ronna; toutefois, dans la partie français-italien il n'y a pas d'indications pour reconnaître le genre des noms géographiques en français.

\subsection{Les aspects formels}

Ces dictionnaires qui, tous trois, ont pour objectif de contenir la matière la plus vaste possible dans un format réduit et de s'adresser à un grand nombre d'étudiants et de lecteurs, offrent des caractéristiques formelles quasiment identiques, à l'enseigne d'une lisibilité médiocre : la dimension du caractère est très réduite, il n'y a pas d'interligne majeure entre les diverses entrées et dans presque toutes les éditions, il y a trois colonnes par page sauf pour le Ronna où les lemmes sont disposés sur deux colonnes. Dans les trois cas, la consultabilité, elle aussi, s'avère plutôt compromise : l'entrée n'est pas en gras (seulement l'initiale est majuscule), la catégorie grammaticale et le traduisant sont tous deux en italique, la composition est en débord; une partie du mot continue à la ligne suivante ou à celle précédente et les deux parties sont unies par des crochets. Quant au poids et aux prix des ouvrages, ils sont tous trois très contenus : ils varient de 500 grammes pour le Biagioli-Ronna aux 200 grammes pour le Barberi-Ronna et le Ronna. Les prix sont d'environ 5 francs, sauf pour le Barberi-Ronna dont le prix varie entre 3 et 6 francs pour l'édition brochée et entre 4 et 7 francs pour l'édition reliée façon maroquin. Les aspects formels respectent donc les caractéristiques des dictionnaires d'apprentissage de l'époque ( $c f$. $\$ 3.3$ ).

\subsection{Les paratextes}

En plus de l'avant-propos et de la table des abréviations (fournies dans les deux langues), à partir de l'édition de 1838, l'usager trouve dans le Biagioli-Ronna la conjugaison des verbes italiens et les signes conventionnels utilisés à l'intérieur de la microstructure.

Le dictionnaire donne en outre, dans les deux langues, à la fin de chacune des deux parties, la " Table des noms d'hommes et de femmes les plus usités; contenant aussi les noms des Dieux, des Princes, etc. qui se rencontrent fréquemment dans les livres » et le "Dictionnaire géographique contenant les noms de nations, royaumes, provinces, villes, fleuves, etc. qui ne s'écrivent pas de même en Français et en Italien ».

Dans l'avant-propos, Ronna déclare n'être pas « avare d'explications grammaticales » et il en est vraiment ainsi : la table de conjugaison des verbes italiens et les explications sur le genre des noms dans le dictionnaire géographique sont en effet très riches.

Le Barberi-Ronna présente l'avant-propos, non signé, non daté, de Barberi, et, dans la première partie, la «Table de quelques noms propres d'hommes, de femmes, de pays, de nations " et les abréviations employées dans ce dictionnaire; dans la seconde, la liste de quelques noms propres d'hommes, de femmes, de pays, de nations et les abréviations employées dans ce dictionnaire. Le Barberi-Ronna ne contient pas de tables consacrées à la conjugaison des verbes et il apparaît plutôt pauvre sur le plan paratextuel. 

des abréviations (dans les deux langues) et les signes conventionnels utilisés à l'intérieur de la microstructure. Les signes graphiques pour indiquer la prononciation de l'italien sont placés à la fin de la liste des abréviations (cf. § 3.8.3). Le Dictionnaire n'a pas de pages consacrées à la conjugaison des verbes italiens comme dans le BiagioliRonna, quoique l'éditeur énumère, parmi les qualités du dictionnaire, la présence d'explications grammaticales. À la fin de chacune des parties on trouve la liste des «noms propres de personne, historiques et mythologiques plus usités dans les livres » et un « Dictionnaire géographique ».

\subsection{Les sources} lexicographiques et grammaticales auxquelles Ronna a puisé. avoir revu le dictionnaire «après avoir compulsé les meilleurs Dictionnaires ItaliensFrançais » et les «importants travaux de Biagioli, auteur d'une excellente grammaire élémentaire italienne ». Les travaux de Biagioli - écrit-il encore dans l'avant-propos " nous offraient une mine précieuse, nous y avons puisé et nous nous faisons un devoir de payer à la mémoire du savant philologue le tribut de notre admiration et de notre reconnaissance ». Enfin les pages sur la conjugaison des verbes italiens constituent « un extrait de la théorie des verbes italiens rédigée sur les ouvrages de Cinonio, Mastrofini, Pistolesi ». D'autres sources lexicographiques sont la Nouvelle édition du Dictionnaire de l'Académie et celle "du Dictionnaire de la langue italienne publié à Bologne de 1819 à $1826 »^{9}$. Pour la rédaction du Barberi-Ronna, outre à l'Académie de la Crusca explicitement citée, la source lexicographique à laquelle se réfère l'auteur est Le Grand Dictionnaire d'Alberti, qu'il considère comme un exemple de dictionnaire bien fait, tandis qu'il critique âprement Cormon et Manni, Bottarelli et Martinelli. Pour la rédaction du Ronna, les sources lexicographiques sont le Dictionnaire de l'Académie française et « les meilleurs dictionnaires publiés en Italie».

\subsection{La nomenclature}

La nomenclature des trois dictionnaires est ordonnée alphabétiquement et contient des unités lexicales simples, des unités lexicales pluri-verbales, des séquences figées, des exemples avec un verbe à l'infinitif et conjugué, des collocations et des locutions. Dans le Barberi-Ronna et dans le Ronna les noms propres de personne, historiques, mythologiques et géographiques qui ne s'écrivent pas de la même façon en français et en italien sont regroupés dans une liste à part. Nous avons trouvé des formes parémiologiques seulement dans le Barberi-Ronna: "per un punto Martin perse la cappa » (« Pour un point Martin perdit son âne »).

Quoiqu'il s'agisse de trois dictionnaires foncièrement semblables, il est possible de mettre en évidence quelques particularités les caractérisant.

Premièrement, le traitement des lettres ramistes n'est pas homogène : en effet, dans la partie italien-français du Biagioli-Ronna, le J n'est pas considéré comme une lettre à part, mais est inséré à la fin de la lettre I, avant les trois derniers mots commençant par $i$; dans le Barberi-Ronna I et $\mathrm{J}$ sont unifiés dans la partie française ; $j$ est réduit à $i$ dans 
la partie italien-français ; enfin, dans le Ronna, le J est une lettre à part et le nombre de lemmes qui commencent par $j$ est supérieur à celui du Biagioli-Ronna, car quelques mots commençant par la semi-voyelle $j$ y ont été insérés. En ce qui concerne $U$ et $V$, ils sont unifiés dans les deux parties du Barberi-Ronna, et séparées dans les autres dictionnaires.

Deuxièmement, l'on remarque des différences dans le traitement de la lettre $\mathrm{K}$ : dans le Biagioli-Ronna, le $\mathrm{K}$ suit la lettre I, mais contient une seule entrée, la lettre $\mathrm{K}$ ellemême : « K, sm. Lettera detta kappa, non è necessaria agl'Italiani, e viene solo adoperata in qualche nome straniero $»^{10}$; dans le Ronna la situation est la même, mais la définition est ici enrichie par des indications graphématiques : «cette lettre n'est pas usitée dans la langue italienne ; elle est remplacée par $\mathrm{C}$ ou $\mathrm{CH} »$.

Troisièmement, le traitement des mots triviaux se révèle assez intéressant par rapport aux déclarations faites par Ronna ou par l'éditeur. En effet, dans le Biagioli-Ronna, Ronna déclare avoir inséré "tous les mots autorisés par l'usage moins toutefois quelques-uns mal sonnant à l'oreille chaste de la jeunesse », cependant leur traitement manque de cohérence. Dans la liste des abréviations, en effet, n'apparaissent pas les marques populaire, vulgaire, trivial, mais les entrées CUL, MERDE, MERDEUX, SALOPE sont présentes, sans aucune marque. Le mot SALOPE est traduit par «lercia, donna disonesta » ("femme sale, malhonnête »), tandis que dans la partie italien-français, on trouve " TRÔJA sf. truie; (fig.) salope, f.". Le traitement des mots triviaux est le même dans le Ronna, alors que dans le Barberi-Ronna il n'y a pas de déclarations à propos des façons de dire erronées, peu élégantes ou vulgaires : on ne trouve pas les marques pop., vulg., triv. dans la table des abréviations, cependant CUL, MERDE figurent parmi les lemmes et sont sans marques de registre.

\subsection{La prononciation}

Dans le Biagioli-Ronna les indications sur la prononciation concernent exclusivement l'italien. On indique l'accent tonique sur tous les mots dans l'ordre alphabétique, et sur ceux de prononciation difficile (mot avec accent tonique sur l'antépénultième) dans le corps de l'ouvrage.

Pour distinguer $e$ et $o$ ouverts on utilise l'accent circonflexe. L'indication du signe graphique utilisé ne se trouve pas dans le paratexte, à la fin de la liste des abréviations, comme pour les autres signes graphiques utilisés à l'intérieur de la microstructure. La lettre $\mathrm{H}$ dans la partie français-italien est marquée d'une note en bas de page dans laquelle on explique que les guillemets apicaux indiquent le $h$ aspiré, mais non dans la partie italien-français devant le traduisant. Dans la partie italien-français, la lettre $\mathrm{H}$ contient cette précision :

Cette lettre ne se fait pas sentir dans la prononciation italienne et ne s'aspire jamais; ce caractère n'est employé que dans ces quatre formes du verbe avere, ho, hai, ha, hanno, j'ai, tu as il a, ils ont ; pour ne pas les confondre avec les mots o, ou, ai, aux, $a$, à, anno, année. On l'emploie aussi comme lettre auxiliaire pour rendre le son $\mathrm{du} \mathrm{c}$ et du $\mathrm{g}$ dur avant les voyelles $\mathrm{i}$ et $\mathrm{e}$ et enfin dans quelques interjections, où il sert à soutenir le son de la voyelle.

Le paratexte du Barberi-Ronna ne fournit pas d'indications sur la prononciation, mais l'accent tonique est systématiquement indiqué sur les mots italiens. Le $h$ aspiré n'est pas signalé, de même qu'aucune particularité de la prononciation française. À la lettre 
$\mathrm{H}$ dans la partie italien-français sont contenues des indications phonétiques et orthographiques :

[...] elle ne sert que pour rendre le son $\mathrm{du} g$ et du $c$ devant les voyelles, $e, i$, et pour distinguer, dit-on, ho, hai, ha, hanno de o, ai, a, anno. On l'emploie dans quelques interjections auxquelles elle donne une certaine aspiration, et un son plus prolongé et plus soutenu.

Dans le Ronna, l'indication de la prononciation n'est pas systématique ; elle n'est pas fournie dans les deux langues, mais seulement pour l'italien; elle ne donne donc pas, comme dans les autres dictionnaires, toutes les indications de prononciation qui pourraient résoudre les difficultés pour un francophone, mais en donne seulement certaines : « ̂े » indique $e$ ouvert ; «é », $e$ fermé ; « ô », o ouvert ; «ó », o fermé ; « z », $z$ dur, « $z », z$ doux; " ' «, accent de prosodie. Par rapport au dictionnaire Biagioli-Ronna, les signes graphiques pour indiquer la prononciation ont augmenté. Enfin, le traitement de la lettre $\mathrm{H}$ est le même que dans le Biagioli-Ronna.

\subsection{Les entrées}

Le Biagioli-Ronna et le Ronna présentent une microstructure semblable. Toutes les entrées sont suivies de l'indication de la catégorie grammaticale, du genre des substantifs, du féminin des adjectifs. Dans le Biagioli-Ronna les substantifs et les adjectifs épicènes sont signalés par le numéro $2:$ «IRREPARABILE, 2. a. irréparable ». Dans les deux dictionnaires, les pluriels irréguliers sont indiqués, mais pas toujours de façon cohérente: "CAPARRA sf. arrhes sf. plur. et ARRHES sf. plur. caparra sf.»; "STEMMA, sm. armoiries, f. pl. mais : ARMOIRIES, sf. Pl. arme, f. pl. scudo gentilizio, m. » (la traduction ne correspond pas à l'entrée dans la partie italien-français).

Les deux dictionnaires présentent des discriminateurs de sens : «ASOLO, sm, respiration, $f$. (per occhiello) boutonnière, $f$.»; le Biagioli-Ronna est cependant plus riche sous cet aspect :

PEINE, sf. tormento, m. pena, penalità ; ripugnanza, $\mathrm{f}$.

- d'esprit, ansietà, $\mathrm{f}$.

- d'un artisan, salario d'un lavorante, $m$.

Le signe « - » indique la répétition du vocable qui précède par ordre alphabétique aussi bien dans le Biagioli-Ronna que dans le Ronna. Les acceptions sont séparées par un point virgule et les synonymes par une virgule : «BLÉ grano, frumento » mais « AUDITEUR uditore ; ascoltatore, scolare $»^{11}$.

Aucun signe graphique n'est utilisé pour signaler les archaïsmes. Les marques de registre (familier, de langages sectoriels, de connotation) sont peu nombreuses et n'augmentent pas d'une édition à l'autre; elles sont encore moins nombreuses dans le Ronna, où manquent par ailleurs les marques de langages sectoriaux et de registre familier qui sont présentes dans le Biagioli-Ronna.

Des variantes morphologiques (MISURAMENTO, MISURAZIONE) ou orthographiques (TRISTEZZA, TRISTIZIA ; TROGOLO, TRUOGOLO, TRUOGO ; TRIONFARE, TRIUNFARE) sont présentes dans toutes les éditions.

On fournit des gloses descriptives ou de définition: " CARBONERIA, sf. (società segreta avente per iscopo l'indipendenza dell'Italia), carbonerie, $f . »^{12}$. " OSSIGENE, sm. (sostanza che genera l'acidità in tutti i corpi con cui si combina), oxygène, $m$. $»^{13}$. Il importe de remarquer que la glose n'est pas fournie dans la partie français-italien; en outre, les 
gloses descriptives du Ronna sont moins nombreuses que dans le Biagioli-Ronna, surtout dans la partie italien-français : elles manquent, par exemple, pour METAMORFOSI (« métamorphose »), METEMPSICOSI (« métempsycose »), ossigenE (« oxygène »). d'affinités avec les travaux lexicographiques auxquels il s'attelait à la même époque : ni les abréviations, ni la nomenclature ni mêmes les tables des conjugaisons ne correspondent dans les deux types d'ouvrages. Ronna considérait sans doute le Guide comme un gagne-pain et il ne fit que reprendre ou s'inspirer, pour le rédiger, d'autres ouvrages similaires, ou peut-être même les traduire; nous ne pouvons pas en l'état actuel démontrer cette hypothèse qui requerrait de plus amples enquêtes sur l'ensemble des éditions des Guides.

Par contre, en ce qui concerne les dictionnaires, il voulut y consacrer un effort supplémentaire : en particulier, la reprise du Dictionnaire de Biagioli, qui lui fut confié par Hingray, est le résultat d'un travail constant et prolongé d'amélioration par rapport à la reprise du Dictionnaire de Barberi, publié la même année, à la suite d'une révision plutôt sommaire. Enfin, pour ce qui concerne son propre Dictionnaire, Ronna reste très lié à son remaniement du Biagioli, qui constitue sans aucun doute l'étalon dans son travail de lexicographe. 
ailleurs, les éléments biographiques de Ronna nous permettent de supposer que ce professeur engagé devait accorder une plus grande attention à la littérature tout en étant bien conscient que c'est par l'enseignement de la langue qu'il réussissait à pourvoir aux besoins de sa famille. Quoique ses anthologies soient essentiellement destinées à un public d'apprenants, Ronna donnait la plus grande importance à la diffusion de la littérature italienne en France car c'est par les lettres qu'il souhaitait sans doute que les idéaux du Risorgimento atteignent le milieu intellectuel français ${ }^{15}$. Ce patriote amateur des belles lettres ne fut donc pas un novateur ni en tant que lexicographe ni en tant qu'auteur de manuels, mais, par la diffusion de ses ouvrages (Lillo 2008: passim), il contribua avec Cormon et Manni, Morlino et de Roujoux, Briccolani, Caccia et bien d'autres encore, à créer la grande tradition lexicographique bilingue franco-italienne au XIX ${ }^{\mathrm{e}}$ siècle avant que les dictionnaires de Candido Ghiotti ne viennent s'imposer au tournant du XXe siècle et bien au-delà (Merger 2013 : 115).

\section{BIBLIOGRAPHIE}

\section{Sources primaires}

RONNA, Antonio \& BARBERI, Joseph-Philippe (1836). Dictionnaire français-italien composé sur les meilleurs dictionnaires français et italiens, contenant plus de 10000 mots omis dans tous les autres dictionnaires portatifs : par J.-Ph. Barberi, revu et augmenté d'explications grammaticales, par A. Ronna. Paris : Baudry [et éditions de 1842, 1843, 1847, 1850].

RONNA, Antonio \& BIAGIOLI, Giosafatte (1836). Dictionnaire français-italien et italien-français à l'usage des maisons d'éducation des deux nations, rédigé sur les travaux de feu G. Biagioli auteur des commentaires historiques et littéraires sur Dante, Pétrarque, etc. etc., d'après la nouvelle édition du Dictionnaire de l'Académie française et de celle du Dictionnaire de la langue italienne, publié à Bologne de 1819 à 1826, plus complet qu'aucun autre Dictionnaire abrégé, par A. Ronna. Paris : Hingray [et éditions de 1838, 1839, 1840, 1841, 1843, 1846, 1848, 1851, 1855, 1859, 1860, 1897].

RONNA, Antonio (1837). Teatro scelto italiano. Commedie, drammi, tragedie tratte da : Goldoni, Albergati, Sografi, De Rossi, Giraud, Nota, Metastasio, Alfieri, Monti, Manzoni, Niccolini, con note biografiche. Paris : Baudry.

RONNA, Antonio (1840). Guide de la conversation français-italien, à l'usage des Voyageurs et des Étudiants, par A. Ronna, comprenant un vocabulaire des mots usuels, des conjugaisons appliquées, des phrases familières et élémentaires, des dialogues, des idiotismes, proverbes, des modèles de lettres, billets, la concordance des monnaies, poids et mesures de France et d'Italie. Paris : Hingray [et éditions de 1840, 1843, 1853, 1855, 1859, 1861, 1864, 1873].

RONNA, Antonio (1841). Lectures italiennes, Cours supérieur comprenant : Tasse, Jérusalem délivrée chants XI et XII, Dante, premier et troisième chants de l'Enfer, Manzoni, Inni sacri. Avec les notes explicatives de M. Biagioli et de M. Ronna, auteur du Dictionnaire. Paris : Hingray. 
RONNA, Antonio (1843). Gemme o rime di poetesse italiane antiche e moderne, scelte da A. Ronna. Paris : Baudry.

RONNA, Antonio (1853a). Études sur la littérature italienne. Giusti. Paris : Impr. de Pillet fils aîné. RONNA, Antonio (1853b). Novelline per uso de' fanciulli e de' giovinetti scritte da A. Ronna e da altri. Paris : Truchy.

RONNA, Antonio (1858). Dictionnaire français-italien contenant les mots admis par l'Académie française et par les meilleurs Dictionnaires publiés en Italie, les termes de science, d'arts, de commerce, ceux employés dans la navigation à la vapeur, et les chemins de fer, etc. avec l'indication des genres dans les deux langues, l'accent tonique dans la partie italienne et les différentes prononciation de l'E, de l'O et du Z, etc. etc. etc. par A. Ronna, auteur du dictionnaire français-italien et italien-français à l'usage de la jeunesse, etc. etc. etc. Paris : Hingray [et éditions de $1861,1862,1864,1883]$.

RONNA, Antonio (1862). Favole di Antonio Ronna. Paris : Truchy.

\section{Sources secondaires}

Biographie universelle ancienne et moderne (1835). Supplément : t. LVIII. Paris : Michaud. BONOLA, Ferdinando (1869). I patrioti italiani : storia e biografia, vol. $\mathrm{I}^{\mathrm{er}}$. Milan : Giocondo Messaggi, Tipografo librajo-editore.

CоLомво, Angelo (2007). «I lunghi affanni ed il perduto regno ». Cultura letteraria, filologia e politica nella Milano della Restaurazione. Besançon : Presses Universitaires de Franche-Comté.

CRISTIANO, Flavia (1986). L'antiquariato librario in Italia : vicende, protagonisti, cataloghi. Rome : Gela editrice.

Dizionario biografico degli Italiani (1960). Rome : Istituto della Enciclopedia Italiana fondata da Giovanni Treccani.

FONZI COLUMBA, Maria Adelaide (1972). « L'emigrazione ». In Bibliografia dell'età del Risorgimento in onore di Alberto M. Ghisalberti, vol. 2. Florence : Olschki, 425-469.

LILLO, Jacqueline (2006). « Les dictionnaires scolaires bilingues français/italien ». In Nadia Minerva

(éd.). Lessicologia e lessicografia nella storia degli insegnamenti linguistici. Bilanci e prospettive. Bologne : CLUEB, 221-236.

LILlo, Jacqueline (éd.) (2008). 1583-2000 : Quattro secoli di lessicografia italo-francese. Repertorio analitico dei dizionari bilingue. Berne : Peter Lang.

MADONIA, Francesco Paolo Alexandre (2016). « Le Dictionnaire de l'Académie française source des dictionnaires bilingues franco-italiens (1694-1870). In Gabriel de Broglie, Hélène Carrère d'Encausse, Giovanni Dotoli, Mario Selvaggio (éds). Le Dictionnaire de l'Académie française. Langue, Littérature, Société, Rome : Edizioni Universitarie Romane, 163-177.

MARTIN Henri-Jean, CHARTIER Roger, VIVET Jean-Pierre (1985). Histoire de l'édition française, t. III, Le temps des éditeurs. Du Romantisme à la Belle Époque. Paris : PROMODIS.

MERGER, Marie-France (2013). « Deux dictionnaires de Candido Ghiotti qui ont marqué le XX ${ }^{\mathrm{e}}$ siècle ». In Jacqueline Lillo (éd.). Les best-sellers de la lexicographie franco-italienne. XVI ${ }^{e}$-XXI ${ }^{e}$ siècle. Rome : Carocci, 115-133. 
MUEL, Francine (1980). «L'alphabétisation de Calvin à Jules Ferry. Note critique ». Annales. Économies, Sociétés, Civilisations, 1, 81-91.

MUNIER, Bertrand (2003). 1791-2003. Le grand livre des élus vosgiens. Conseillers généraux et régionauxdéputés - sénateurs - ministres. Haroué : Gérard Louis éditeur.

PALAZzolo, Maria Iolanda (1987). « Un editore francese in lingua italiana : Louis Claude Baudry ». Studi storici, XXVIII, 1, 203-224.

PROVENZAL, Aristide (1867). « Antonio Ronna. Necrologia ». Rivista bolognese di scienze, lettere ed arti e scuole, vol. II, 328-337.

PRUVOST, Jean (2001). « Les dictionnaires d'apprentissage monolingues de la langue française (1856-1999). Problèmes et méthodes ». In Jean Pruvost (éd.). Les dictionnaires de langue française. Dictionnaires d'apprentissage. Dictionnaires spécialisés de la langue. Dictionnaires de spécialité. Paris : Honoré Champion, 67-95.

RIUS DALMAU María Inmaculada (2009). « L'enseignement/ apprentissage multilingue dans le contexte culturel européen de la première moitié du XIXe siècle : à propos de la Grammaire polyglotte de Simon Jost (1840) ». Documents pour l'histoire du français langue étrangère ou seconde, 42, 127-148.

\section{NOTES}

1. Sur ce général espagnol d'origine française, appartenant probablement à la même famille que celle du plus célèbre maréchal Bessières, voir la notice dans la Biographie universelle ancienne et moderne (1835 : 186-187).

2. Antignano, que l'on ne confondra pas avec Antignano en Piémont, était alors un petit village au sud de Livourne. Au XX $\mathrm{XX}^{\mathrm{e}}$ siècle il est annexé à Livourne et en constitue aujourd'hui un quartier.

3. En particulier: le Teatro scelto italiano (1837) qui, tout en comportant de brèves notices biobiographiques sur les auteurs, n'a pas une vocation ouvertement pédagogique ; les Gemme o rime di poetesse italiane antiche e moderne (1843), qui constituent le tome XLII de la «Biblioteca poetica italiana » dirigée par Antonio Buttura (1770-1832), autre italianisant d'envergure exilé à Paris.

4. Les Études sur la littérature italienne (1853), qui portent essentiellement sur l'analyse de la poésie de Giuseppe Giusti (1809-1850), grand partisan du Risorgimento.

5. «Monnaies des États pontificaux", «Monnaies du Royaume de Naples ». Ces deux tables étaient unies dans les éditions précédentes.

6. En 1840, l'Avis de l'éditeur employait dans la même phrase, de façon plus vague, l'expression: « les règles les plus difficiles du langage».

7. Les exemplaires du Guide français-italien conservés à la BNF sont tous in- $32^{\circ}$. Nous tirons les informations concernant l'édition in $-16^{\circ}$, ainsi que le prix des deux formats, de la quatrième de couverture de l'édition de 1855.

8. La comparaison entre les différentes éditions de tous les dictionnaires cités a été faite à partir des données recueillies dans le Repertorio analitico dei dizionari bilingui (Lillo : 2008), auquel nous avons contribué avec des fiches concernant les dictionnaires d'Antonio Ronna.

9. Il s'agit du Gran Dizionario della lingua italiana, Bologne, Masi, 1819-1826, 7 volumes in-4 $4^{\circ}$ dont les principaux auteurs furent Paolo Costa, Francesco Cardinali et Francesco Orioli.

10. «Lettre dénommée kappa, non nécessaire aux Italiens; utilisée seulement dans quelques noms étrangers ».

11. « scolare » : « écolier» 
12. « société secrète ayant pour but l'indépendance de l'Italie ».

13. "substance qui génère l'acidité dans tous les corps avec lesquels elle se combine ».

14. Dans le Biagioli-Ronna on lit par exemple: "Lo (articolo mascolino avanti le parole principiate da s seguita da altra consonante, o da $\mathrm{z}$, ed avanti le vocali), le, Lo specchio, Lo zio, le miroir, l'oncle, l'amour, l'aurore » (" article masculin devant les paroles commençant par $s$ suivi d'une autre consonne, ou par $z$, et devant les voyelles ») ; « Lei pron (femminino de' casi obbliqui d'ella), elle, di lei, a lei da lei, d'elle, à elle » (« féminin des cas obliques de ella »); alors que dans le Ronna : « Lo, art. m. le ; lo specchio, lo zio, l'amore, le miroir, l'oncle, l'amour » et « Lei, pron. (on l'emploie dans la conversation comme sujet), elle, di lei, a lei, da lei, d'elle, à elle ».

15. Les avis de l'auteur, quelques notes éparses, les morceaux choisis et l'étude littéraire sur Giusti démontrent sans conteste le souci de prôner un message politique.

\section{RÉSUMÉS}

Antonio Ronna (1801-1866), lexicographe et professeur, représente un de ces exemples d'intellectuel engagé qui participèrent au Risorgimento et s'exilèrent pour ne pas se plier au joug du régime autrichien. En 1836, il complète, grâce aux fiches laissées par Biagioli, le Dictionnaire italien-français, français-italien: c'est le début d'une activité qui en fera l'un des lexicographes à grand succès du XIX ${ }^{\mathrm{e}}$ siècle. La même année, à la suite d'une révision plutôt sommaire, il publie aussi une reprise du Dictionnaire de Barberi; il faudra par contre attendre 1858 pour que son propre Dictionnaire, inspiré de celui de Biagioli, voie le jour. À l'activité de lexicographe, Ronna joint celle de professeur : l'enseignement de la langue italienne dans un souci de rapprochement constant avec la langue française lui donnera l'envie d'écrire des ouvrages pédagogiques, parmi lesquels le Guide de la conversation français-italien (1840 et éditions successives jusqu'en 1873) se taille la part du lion. Les deux voies de la production pédagogique de Ronna semblent toutefois ne pas se croiser: si l'on excepte la reprise du Dictionnaire de Biagioli et son propre Dictionnaire, l'auteur semble nourrir un intérêt modeste envers ses ouvrages pédagogiques, qui lui servent essentiellement de gagne-pain.

The lexicographer and teacher Antonio Ronna (1801-1866) is one of those examples of committed intellectuals who took part in the Risorgimento and left their countries not to bend to the Austrian regime. In 1836, thanks to the records left by Biagioli, he completed the Dictionnaire italien-français, français-italien : this was the beginning of an activity that would make him one of the best-known lexicographers of the $19^{\text {th }}$ century. In the same year, after a quite brief revision, he resumed the Dictionary of Barberi and published it. It was only in 1858 that, inspired by Biagioli's works, he published his own dictionary. Not only was Ronna a lexicographer, but also a teacher: his teaching the Italian language together with his constant interest for the French language led him to write educational books, including the Guide de la conversation français-italien (1840 and further editions until the 1873 edition) which is his most important work in this field. Ronna paid great attention to his lexicographical works, mainly Biagioli's and his own, and had little interest in the other pedagogical works that he published only to earn his living. 
INDEX

Mots-clés : lexicographie bilingue français/italien, guide de conversation, anthologies, Risorgimento, histoire de l'enseignement de l'italien en France

Keywords : French/Italian bilingual Lexicography, Phrasebook, Anthology, Risorgimento, History of teaching of Italian in France

\section{AUTEUR}

\section{FRANCESCO PAOLO ALEXANDRE MADONIA}

Université de Palermefrancescopaolo.madonia@unipa.it 